\title{
Regional Differences in Specific Health Examination Utilization and Medical Care Expenditures in Japan
}

\author{
Nozomu Mandai1 ${ }^{*}$, Mayumi Watanabe ${ }^{1,2}$ \\ ${ }^{1}$ Ibaraki Prefectural University of Health Sciences, Ibaraki, Japan \\ ${ }^{2}$ Faculty of Science and Engineering, Chuo University, Tokyo, Japan \\ Email: *mandain@ipu.ac.jp
}

How to cite this paper: Mandai, N. and Watanabe, M. (2020) Regional Differences in Specific Health Examination Utilization and Medical Care Expenditures in Japan. Health, 12, 1143-1150.

https://doi.org/10.4236/health.2020.129084

Received: August 14, 2020

Accepted: September 15, 2020

Published: September 18, 2020

Copyright $\odot 2020$ by author(s) and Scientific Research Publishing Inc. This work is licensed under the Creative Commons Attribution International License (CC BY 4.0).

http://creativecommons.org/licenses/by/4.0/ (c) (i) Open Access

\begin{abstract}
Background: Despite having one of the most successful health systems in the world, annual medical expenditures in Japan have been increasing year to year. We sought to clarify regional differences in medical expenditures by analyzing the relationship between the specific health examination coverage and medical care expenditure by prefecture of Japan. Methods: We used data from the National Database of Health Insurance Claims and Specific Health Checkups (NDB) Open Data Japan (2015) and Overview of 2015 National Medical Expenses to compare medical care expenditure per capita and proportions of persons receiving specific health examination between Japan nationally and individual prefectures. Results: National medical expenditures were 42.3 trillion Japanese yen (JPY) (3851 hundred million dollars), with a national per capita rate of JPY347,219 (USD3156). Per capita medical expenditure rates by prefecture ranged from JPY290,900 (USD2645) in Saitama Prefecture to JPY 444,000 (USD4036) in Kochi Prefecture. The proportion of persons receiving specific health examinations was $49.0 \%$ for Japan overall and ranged from $39.3 \%$ in Hokkaido Prefecture to $63.4 \%$ in Tokyo Prefecture. We observed a significant negative correlation between per capita medical expenditures and the proportion of persons receiving specific health examinations $(\mathrm{R}=0.553, \mathrm{p}<0.001)$. Conclusion: We found a significant negative correlation between per capita medical expenditures and the proportion of persons receiving health examinations: prefectures with lower expenditures tended to have higher rates of medical examinations. Interventions to increase the proportion of persons receiving specific health examinations by prefecture could reduce per capita medical expenditures and reduce prefectural disparities in expenditures.
\end{abstract}




\section{Keywords}

Medical Expenditures, Regional Disparities, Health Examinations

\section{Introduction}

Japan's health system implemented universal health insurance coverage in 1961 and is one of the most successful health systems in the world, despite having one of the highest life expectancies and the highest old-age dependency ratio among advanced economies [1]. However, annual medical expenditures per capita are in-creasing year by year, from JPY267,000 (USD2427) in 2007 to nearly JPY340,000 (USD3091) in 2017 (Figure 1) [2] [3]. To control rising healthcare costs, Japan's Ministry of Health, Labour and Welfare (MHLW) has established an ongoing Medium- and Long-Term Medical Care Expenditure Regulation Plan, which aims to improve prevention of lifestyle diseases and shorten hospital lengths of stay, with an emphasis on joint work between the national and prefectural governments (Figure 2) to address regional differences in costs [4].

Regional and community planning and implementation of health policies have been identified as an important component of maintaining the low costs and improving equity in healthcare [5]. In addition to central policies emphasizing patient-centered health interactions, local efforts tailored to the particular needs of each regional health system are needed to foster equity and address regional

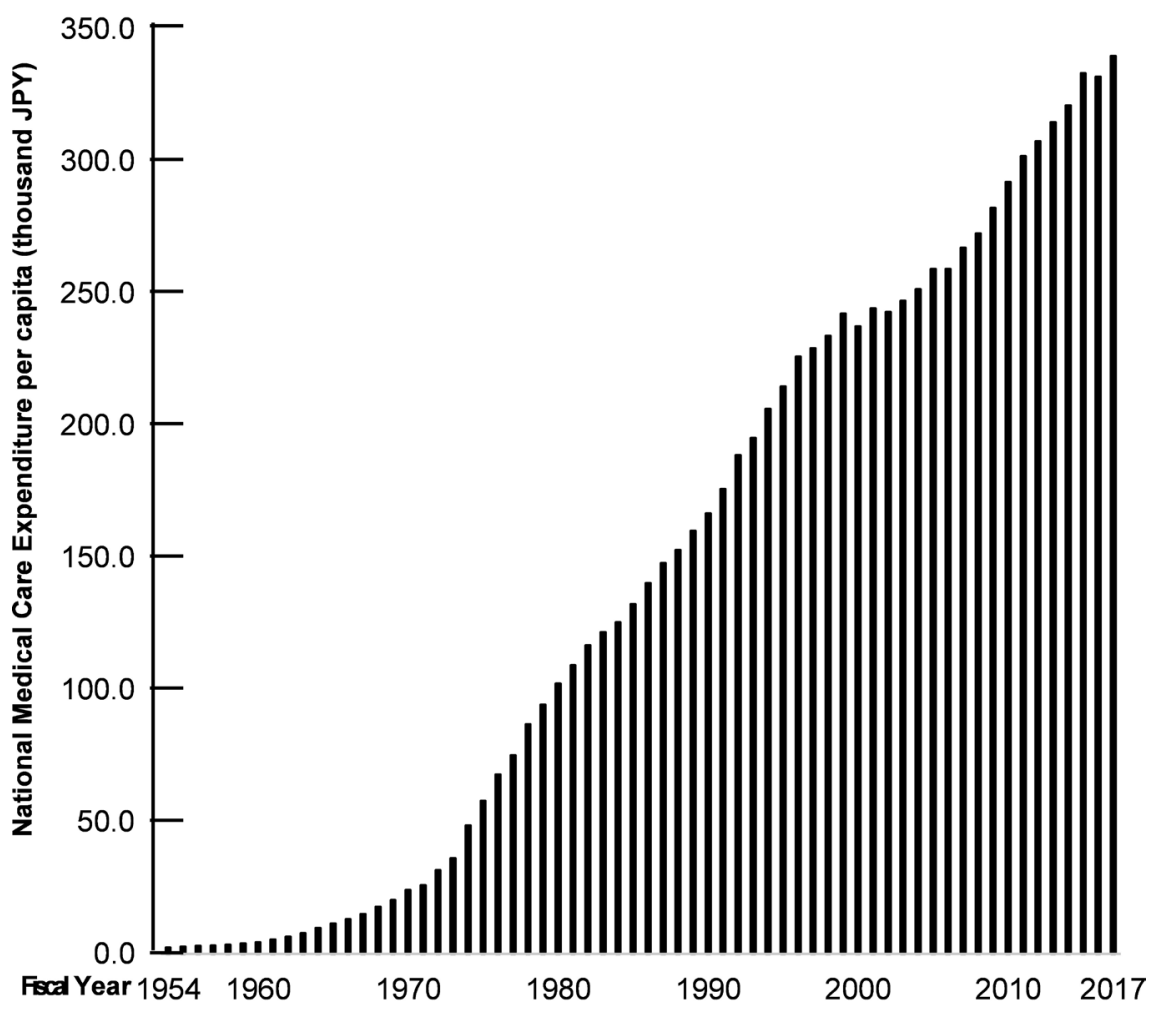

Figure 1. National medical expenditures per capita. JPY: Japanese yen. 


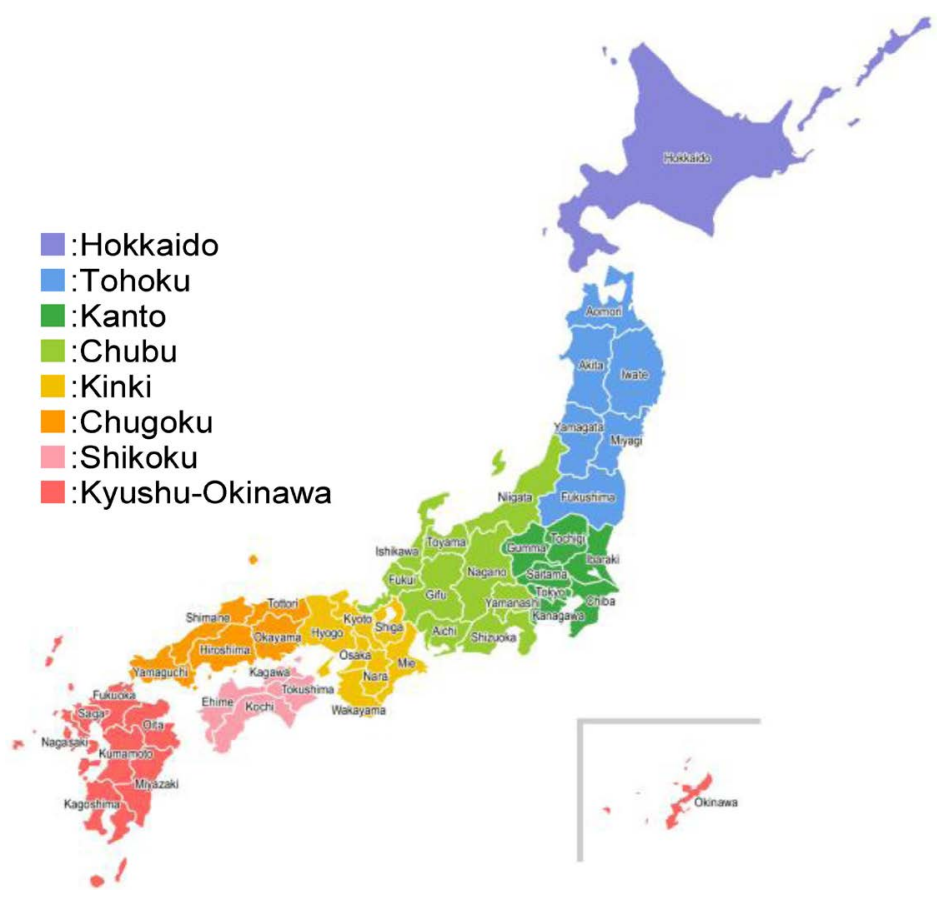

Figure 2. Map of Japan's prefectures. Eight regions: $\square:$ Hokkaido (violet), ๘: Tohoku (blue), $\square$ : Kanto (green), $\square$ : Chubu (light green), $\square$ : Kinki (yellow), $\square$ : Chugoku (orange), $:$ : Shikoku (pink), $\square:$ Kyushu-Okinawa (red).

differences in quality, access, and costs of care. In an effort to further optimize expenditures, the Medium- and Long-Term Medical Care Expenditure Regulation Plan promotes regional difference analysis of medical expenditures.

To clarify regional differences in medical expenditures in Japan, we analyzed the relationship between the specific health examination coverage and medical care expenditure by prefecture of Japan using data from the National Database of Health Insurance Claims and Specific Health Checkups (NDB) Open Data Japan (2015) and Overview of 2015 National Medical Expenses.

\section{Methods}

The NDB was implemented in 2009 by the MHLW in order to plan health policies using national data. Additionally, the database is used for health research purposes. The NDB includes most of the administrative claims and health checkup data from insurers in Japan, covering approximately $98 \%$ of healthcare services in the country. The database contains information on prefecture, sex, age, dates of admission and discharge, and codes for diagnoses, procedures, and prescriptions [6].

Using data from the NDB and the Overview of 2015 National Medical Expenses [7] [8] [9], we compared mean medical care expenditure per capita and mean proportions of persons receiving specific health examinations between Japan nationally and individual prefectures via One Sample t-test. To evaluate the relation-ship between per capita medical expenditures and the proportion of persons receiving specific health examinations, we calculated Pearson correla- 
tion coefficients. A ratio of JPY110: USD1 was used to convert currency (2014 value).

All statistical analyses were performed using the Statistical Package for the Social Sciences (SPSS) version 26.0 (SPSS, Inc., Chicago, IL, USA). Statistical significance was set at $\mathrm{p}<0.05$. The study was approved by the Medical Ethics Committee of Ibaraki Prefectural University of Health Sciences (approval No. e211-r010620).

\section{Results}

National medical expenditures were 42.3 trillion Japanese yen (3851 hundred million dollars), with a national per capita rate of JPY347,219 (USD3156). Per capita medical expenditure rates by prefecture ranged from JPY290,900 (USD2645) in Saitama Prefecture to JPY444,000 (USD4036) in Kochi Prefecture (Figure 3).

\begin{tabular}{|c|c|c|c|c|}
\hline \multirow{2}{*}{ Prefecture } & \multicolumn{2}{|c|}{ Total (100 mio) } & \multicolumn{2}{|c|}{ per capita (thousands) } \\
\hline & JPY & USD & JPY & USD \\
\hline Japan & 423644 & 3851 & 347.2 & 3.15 \\
\hline Hokkaido & 21184 & 193 & $393.6 * *$ & $3.58 * *$ \\
\hline Aomori & 4469 & 41 & 341.7 & 3.11 \\
\hline Iwate & 4145 & 38 & $323.8^{* *}$ & $2.94 * *$ \\
\hline Miyagi & 7221 & 66 & $309.4 * *$ & $2.81 * *$ \\
\hline Akita & 3751 & 34 & $366.6 * *$ & $3.33 * *$ \\
\hline Yamagata & 3821 & 35 & 340.0 & 3.09 \\
\hline Fukushima & 6335 & 58 & $331.0 *$ & $3.01 *$ \\
\hline Ibaraki & 8826 & 80 & $302.6 * *$ & $2.75 * *$ \\
\hline Tochigi & 6008 & 55 & $304.3^{* *}$ & $2.77 * *$ \\
\hline Gunma & 6260 & 57 & $317.3 * *$ & $2.88^{* *}$ \\
\hline Saitama & 21139 & 192 & $290.9 * *$ & $2.64 * *$ \\
\hline Chiba & 18118 & 165 & $291.1 * *$ & $2.65 * *$ \\
\hline Tokyo & 41433 & 377 & $306.6 * *$ & $2.79 * *$ \\
\hline Kanagawa & 27186 & 247 & $297.9 * *$ & $2.71 * *$ \\
\hline Niigata & 7110 & 65 & $308.6 * *$ & $2.81 * *$ \\
\hline Toyama & 3557 & 32 & $333.7 *$ & $3.03 *$ \\
\hline Ishikawa & 3993 & 36 & 346.0 & 3.15 \\
\hline Fukui & 2635 & 24 & $334.8^{*}$ & $3.04 *$ \\
\hline Yamanashi & 2772 & 25 & $332.0 *$ & $3.02 *$ \\
\hline Nagano & 6756 & 61 & $321.9 * *$ & $2.93 * *$ \\
\hline Gifu & 6668 & 61 & $328.1 *$ & $2.98 *$ \\
\hline Shizuoka & 11414 & 104 & $308.5 * *$ & 2.80 ** \\
\hline Aichi & 22468 & 204 & $300.3 * *$ & $2.73 * *$ \\
\hline Mie & 5794 & 53 & $319.1 * *$ & 2.90 ** \\
\hline Shiga & 4222 & 38 & $298.8 * *$ & $2.72 * *$ \\
\hline Kyoto & 8994 & 82 & 344.6 & $3.1 \overline{3}$ \\
\hline Osaka & 32193 & 293 & $364.2 *$ & $3.31 *$ \\
\hline Hyogo & 19114 & 174 & 345.3 & 3.14 \\
\hline Nara & 4637 & 42 & 340.0 & 3.09 \\
\hline Wakayama & 3607 & $3 \overline{3}$ & $374.2 * *$ & $3.40 * *$ \\
\hline Tottori & 2000 & 18 & 349.1 & 3.17 \\
\hline Shimane & 2628 & 24 & $378.7 * *$ & $3.44 * *$ \\
\hline Okayama & 6960 & 63 & $362.1 *$ & $3.29 *$ \\
\hline Hiroshima & 10410 & 95 & $366.0 *$ & $3.33 *$ \\
\hline Yamaguchi & 5608 & 51 & $399.2 * *$ & $3.63 * *$ \\
\hline Tokushima & 2968 & 27 & $392.5 * *$ & $3.57 * *$ \\
\hline Kagawa & 3727 & 34 & $381.8 * *$ & $3.47 * *$ \\
\hline Ehime & 5202 & 47 & $375.6 * *$ & $3.41 * *$ \\
\hline Kochi & 3233 & 29 & $444.0 * *$ & $4.04 * *$ \\
\hline Fukuoka & 19353 & 176 & $379.3 * *$ & $3.45 * *$ \\
\hline Saga & 3269 & 30 & $392.5 * *$ & $3.57 * *$ \\
\hline Nagasaki & 5661 & 51 & $411.1 * *$ & $3.74 * *$ \\
\hline Kumamoto & 6954 & 63 & $389.3 * *$ & $3.54 * *$ \\
\hline Oita & 4619 & 42 & $396.2 * *$ & $3.60 * *$ \\
\hline Miyazaki & 4025 & 37 & $364.6^{*}$ & $3.31 \%$ \\
\hline Kagoshima & 6705 & 61 & $406.9 * *$ & $3.70 * *$ \\
\hline Okinawa & 4495 & 41 & $313.5 * *$ & $2.85^{* *}$ \\
\hline
\end{tabular}

Figure 3. Comparison of mean national medical care expenditure per capita between Japan and prefectures. ${ }^{*} \mathrm{p}<0.05$ vs. Japan: ${ }^{* *} \mathrm{p}<0.001$ vs. Japan. JPY: Japanese yen, USD: US dollar. 
The proportion of persons receiving specific health examinations was $49.0 \%$ for Japan overall and ranged from $39.3 \%$ in Hokkaido Prefecture to $63.4 \%$ in Tokyo Prefecture (Figure 4).

We observed a significant negative correlation between per capita medical expenditures and the proportion of persons receiving specific health examinations $(\mathrm{R}=0.553, \mathrm{p}<0.001)$ (Figure 5).

\section{Discussion}

Substantial variation by prefecture exists in per capita medical expenditures and in the proportion of persons receiving specific health examinations. We found a significant negative correlation between per capita medical expenditures and the proportion of persons receiving health examinations: prefectures with lower per capita medical expenditures tended to have higher rates of specific medical

\begin{tabular}{|c|c|c|c|}
\hline Prefecture & $\begin{array}{c}\text { Number of subject pers ons of } \\
\text { specific health examination } \\
\text { (Es timated value) } \\
\mathbf{N}\end{array}$ & $\begin{array}{l}\text { Number of persons } \\
\text { receiving specific } \\
\text { health examinations } \\
\mathbf{N}\end{array}$ & $\begin{array}{c}\text { Proportion of } \\
\text { persons receiving } \\
\text { snecific health } \\
\%\end{array}$ \\
\hline Japan & $53,960,721$ & $27,058,105$ & 49.0 \\
\hline Hokkaido & $2,350,032$ & 922,700 & $39.3 * *$ \\
\hline Aomori & 595,994 & 268,699 & $45.1 * *$ \\
\hline Iwate & 557,451 & 285,497 & $51.2 *$ \\
\hline Miyagi & 974,459 & 561,160 & $57.6 * *$ \\
\hline Akita & 464,384 & 216,038 & $46.5^{*}$ \\
\hline Yamagata & 482,012 & 289,226 & $60.0 * *$ \\
\hline Fukushima & 840,256 & 418,275 & 49.8 \\
\hline Ibaraki & $1,276,424$ & 636,193 & 49.8 \\
\hline Tochigi & 861,134 & 413,788 & 48.1 \\
\hline Gunma & 866,354 & 424,215 & 49.0 \\
\hline Saitama & $3,108,260$ & $1,582,268$ & $50.9 *$ \\
\hline Chiba & $2,645,989$ & $1,400,292$ & $52.9 * *$ \\
\hline Tokyo & $5,470,987$ & $3,466,537$ & $63.4 * *$ \\
\hline Kanagawa & $3,818,126$ & $1,897,594$ & 49.7 \\
\hline Niigata & $1,003,950$ & 538,130 & $53.6 * *$ \\
\hline Toyama & 477,372 & 266,700 & $55.9 * *$ \\
\hline Ishikawa & 500,464 & 272,148 & $54.4 * *$ \\
\hline Fukui & 338,269 & 165,479 & 48.9 \\
\hline Yamanashi & 367,002 & 203,887 & $55.6 * *$ \\
\hline Nagano & 906,675 & 491,303 & $54.2 * *$ \\
\hline Gifu & 896,444 & 439,385 & 49.0 \\
\hline Shizuoka & $1,612,803$ & 852,695 & $52.9 * *$ \\
\hline Aichi & $3,120,031$ & $1,611,190$ & $51.6^{*}$ \\
\hline Mie & 781,152 & 413,973 & $53.0 * *$ \\
\hline Shiga & 577,626 & 287,284 & 49.7 \\
\hline Kyoto & $1,086,395$ & 501,359 & $46.1 * *$ \\
\hline Osaka & $3,729,686$ & $1,700,300$ & $45.6 * *$ \\
\hline Hyogo & $2,366,766$ & $1,101,017$ & $46.5^{*}$ \\
\hline Nara & 597,066 & 253,968 & $42.5^{* *}$ \\
\hline Wakayama & 432,368 & 175,696 & $40.6 * *$ \\
\hline Tottori & 243,173 & 111,613 & $45.9^{* *}$ \\
\hline Shimane & 294,217 & 157,303 & $53.5 * *$ \\
\hline Okayama & 803,606 & 360,190 & $44.8^{* *}$ \\
\hline Hiroshima & $1,208,282$ & 546,760 & $45.3 * *$ \\
\hline Yamaguchi & 610,992 & 256,354 & $42.0 * *$ \\
\hline Tokushima & 330,213 & 153,535 & $46.5^{*}$ \\
\hline Kagawa & 428,981 & 206,545 & 48.1 \\
\hline Ehime & 610,560 & 263,397 & $43.1 * *$ \\
\hline Kochi & 317,589 & 148,141 & $46.6^{*}$ \\
\hline Fukuoka & $2,089,860$ & 946,910 & $45.3 * *$ \\
\hline Saga & 347,651 & 161,586 & $46.5^{*}$ \\
\hline Nagasaki & 596,719 & 261,784 & $43.9 * *$ \\
\hline Kumamoto & 747,187 & 349,147 & 46.7* \\
\hline Oita & 497,130 & 258,714 & $52.0 * *$ \\
\hline Miyazaki & 474,944 & 211,850 & $44.6 * *$ \\
\hline Kagoshima & 693,963 & 334,845 & 48.3 \\
\hline Okinawa & 559,721 & 272,435 & 48.7 \\
\hline
\end{tabular}

Figure 4. Proportion of persons receiving specific health examination by prefecture, fiscal year $2015 .{ }^{*} \mathrm{p}<0.05$ vs. Japan: ${ }^{*} \mathrm{p}<0.001$ vs. Japan. N: number of subjects. 


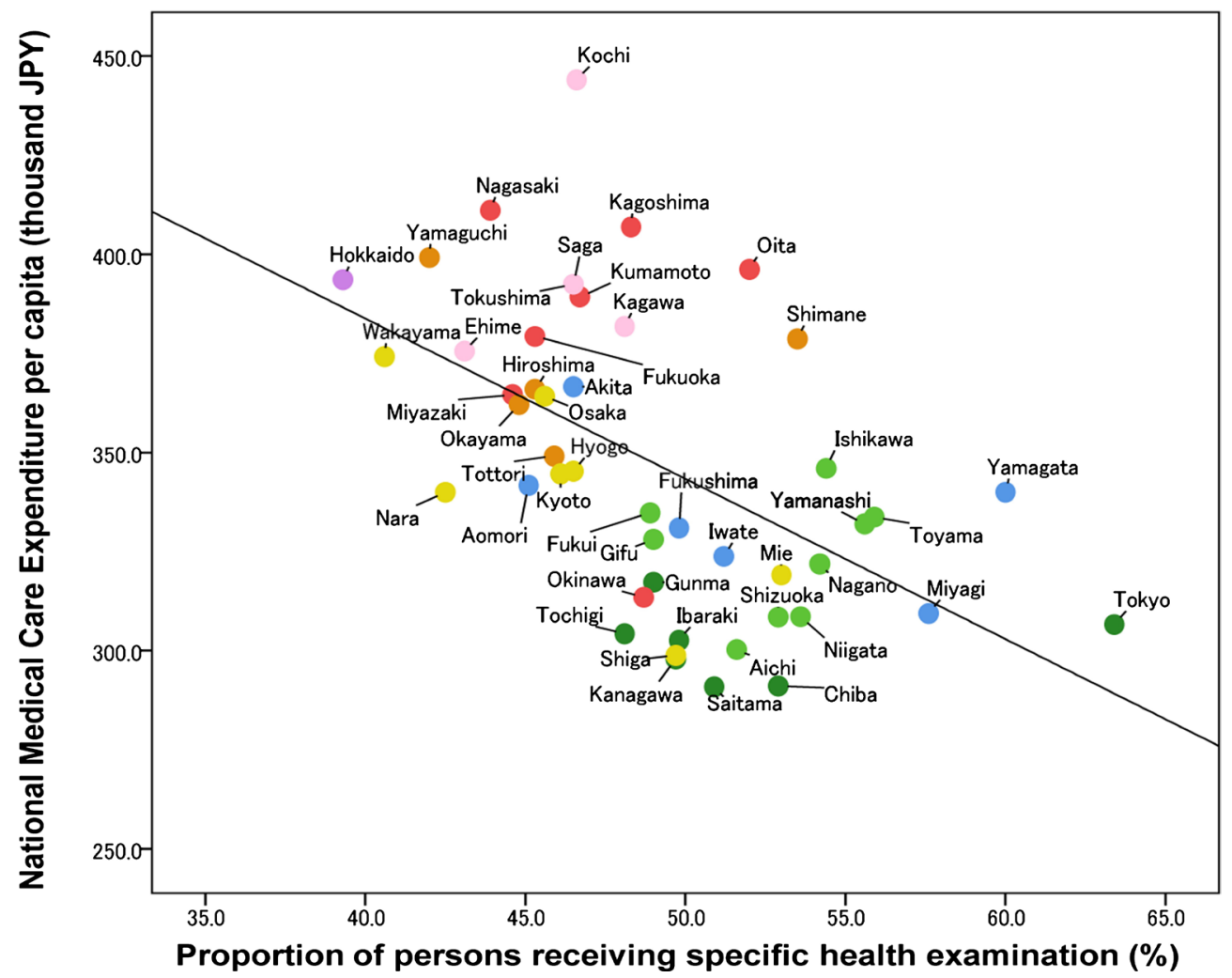

Figure 5. Relationship among national medical care expenditure per capita and proportion of persons receiving specific health examination. JPY: Japanese yen.

examinations.

It is possible that prefectures with high health awareness have high health examination rates and low expenditures. Health literacy is associated with healthy lifestyle, reduced lifestyle disease incidence, and participation in health screening and examinations [10].

Our findings suggest that increasing the proportion of persons receiving specific health examinations by prefecture would have a favorable impact on the medical care expenditure. Low health literacy is associated with less use of preventive services [11] and more unnecessary hospital admissions and emergency department visits [12]. Engagement in specific health examinations could reduce medical expenditures through early detection of risky lifestyle behaviors or lifestyle diseases and other diseases at earlier stages. Less expensive behavioral interventions as primary or secondary prevention can prevent costlier pharmacological interventions required to treat more severe disease. For example, physical inactivity is associated with increased medical expenditures due to increased hospitalizations, physician visits, and medications [13]. Relatively inexpensive lifestyle interventions targeting increased physical activity could prevent future costly treatments of disease sequelae, such as treatment for myocardial infarction related to physical inactivity and obesity. Likewise, lifestyle intervention in pre-diabetes reduces long-term medical expenditures [14], and progression from pre-diabetes to diabetes is associated with one-third higher medical expenditures 
compared to patients who did not progress to diabetes [15].

The strengths of the present study include use of standardized nationwide data on the majority of healthcare services in Japan. The observed correlation between per capita medical expenditures and the proportion of persons receiving specific health examinations is a novel finding and suggests an area for intervention to re-duce medical expenditures and improve population health.

However, some limitations also warrant mention. Data for our analysis was collected 5 years ago, in 2015; an analysis of more recent data is forthcoming. While the NDB covers the vast majority of healthcare expenditures in Japan, some expenditures, such as workplace injuries and injuries covered by automotive insurance, are not included [6]. While administrative claims data are generally regarded as having high validity, they are subject to classification errors and provide limited information on potentially important confounders or covariates [16]. Caution is warranted in interpreting the present results.

\section{Conclusion}

This study suggests that interventions to increase the proportion of persons receiving specific health examinations by prefecture could reduce per capita medical expenditures and reduce prefectural disparities in medical expenditures.

\section{Acknowledgements}

This article was supported in part by a JSPS KAKEN Grant-in-Aid for Early-Career Scientists (N.M. 19K19359).

\section{Ethical Approval}

This study was approved by the Medical Ethics Committee of Ibaraki Prefectural University of Health Sciences (Ibaraki, Japan).

\section{Conflicts of Interest}

The authors declare no conflicts of interest regarding the publication of this paper.

\section{References}

[1] Nozaki, M., Kashiwase, K. and Saito, I. (2017) Health Spending in Japan: Macro-Fiscal Implications and Reform Options. The Journal of the Economics of Ageing, 9, 156-171. https://doi.org/10.1016/j.jeoa.2016.11.002

[2] Japan Ministry of Health, Labour and Welfare (2008) Annual Change in National Medical Care Expenditure per Capita. https://www.mhlw.go.jp/toukei/saikin/hw/k-iryohi/08/toukei1.html

[3] Japan Ministry of Health, Labour and Welfare (2019) Outline of Health, Labour and Welfare Statistics 2019. https://www.mhlw.go.jp/toukei/youran/aramashi/all.pdf

[4] Japan Ministry of Health Law (2009) Medium and Long-Term Medical Care Expenditure Regulation Plan. Ministry of Health, Labour and Welfare, Tokyo. https://www.mhlw.go.jp/english/wp/wp-hw3/dl/2-005.pdf 
[5] Shibuya, K., Hashimoto, H., Ikegami, N., Nishi, A., Tanimoto, T., Miyata, H., et al. (2011) Future of Japan's System of Good Health at Low Cost with Equity: Beyond Universal Coverage. The Lancet, 378, 1265-1273. https://doi.org/10.1016/S0140-6736(11)61098-2

[6] Yasunaga, H. (2019) Real World Data in Japan: Chapter I NDB. Annals of Clinical Epidemiology, 1, 28-30.

[7] Japan Ministry of Health, Labour and Welfare (2015) National Database of Health Insurance Claims and Specific Health Checkups of Japan. https://www.mhlw.go.jp/stf/seisakunitsuite/bunya/0000177221.html

[8] Japan Ministry of Health, Labour and Welfare (2015) Data on the Implementation Status of Specific Health Examination and Specific Health Guidance. https://www.mhlw.go.jp/bunya/shakaihosho/iryouseido01/info02a-2.html

[9] Japan Ministry of Health, Labour and Welfare (2017) Overview of Medical Care Expenditure in 2015. https://www.mhlw.go.jp/toukei/saikin/hw/k-iryohi/15/index.html

[10] Yokokawa, H., Fukuda, H., Yuasa, M., Sanada, H., Hisaoka, T. and Naito, T. (2016) Association between Health Literacy and Metabolic Syndrome or Healthy Lifestyle Characteristics among Community-Dwelling Japanese People. Diabetology \& $\mathrm{Me}$ tabolic Syndrome, 8, 30. https://doi.org/10.1186/s13098-016-0142-8

[11] Wennberg, D.E., Marr, A., Lang, L., O’Malley, S. and Bennett, G. (2010) A Randomized Trial of a Telephone Care-Management Strategy. New England Journal of Medicine, 363, 1245-1255. https://doi.org/10.1056/NEJMsa0902321

[12] Berkman, N.D., Sheridan, S.L., Donahue, K.E., Halpern, D.J., Viera, A., Crotty, K., et al. Health Literacy Interventions and Outcomes: An Updated Systematic Review. Evidence Report/Technology Assessments.

https://www.researchgate.net/profile/Nancy_Berkman/publication/232927322_Heal th_Literacy_Interventions_and_Outcomes_An_Updated_Systematic_Review/links/ 00b49521e417728c60000000/Health-Literacy-Interventions-and-Outcomes-An-Up dated-Systematic-Review.pdf

[13] Pratt, M., Macera, C.A. and Wang, G. (2000) Higher Direct Medical Costs Associated with Physical Inactivity. The Physician and Sportsmedicine, 28, 63-70. https://doi.org/10.3810/psm.2000.10.1237

[14] Dall, T.M., Storm, M.V., Semilla, A.P., Wintfeld, N., O’Grady, M. and Venkat Narayan, K.M. (2015) Value of Lifestyle Intervention to Prevent Diabetes and Sequelae. American Journal of Preventive Medicine, 48, 271-280. https://doi.org/10.1016/j.amepre.2014.10.003

[15] Khan, T., Tsipas, S. and Wozniak, G. (2017) Medical Care Expenditures for Individuals with Prediabetes: The Potential Cost Savings in Reducing the Risk of Developing Diabetes. Population Health Management, 20, 389-396. https://doi.org/10.1089/pop.2016.0134

[16] Virnig, B.A. and McBean, M. (2001) Administrative Data for Public Health Surveillance and Planning. Annual Review of Public Health, 22, 213-230.

https://doi.org/10.1146/annurev.publhealth.22.1.213 